\title{
RNA Origami Nanostructures for Potent and Safe
}

\section{Anti-Cancer Immunotherapy}

Xiaodong Qii, ${ }^{1,2}$, Xiaowei Liu ${ }^{4}$, Lawrence Matiski ${ }^{1,4}$, Ryan Rodriguez Del Villar ${ }^{3,4}$, Theresa Yip ${ }^{2,3}$,

Fei Zhang ${ }^{1,2}$, Sriram Sokalingam ${ }^{4}$, Shuoxing Jiang ${ }^{1,2}$, Li Liu ${ }^{5,6}$, Hao Yan*1,2, Yung Chang*3,4

1 School of Molecular Sciences, Arizona State University, Tempe, AZ 85287, USA.

2 Biodesign Center for Molecular Design and Biomimetics, Biodesign Institute, Arizona State University, Tempe, AZ 85287, USA.

3 School of Life Sciences, Arizona State University, Tempe, AZ 85287, USA.

4 Biodesign Center for Immunotherapy, Vaccines and Virotherapy, Biodesign Institute, Arizona State University, Tempe, AZ 85287, USA.

5 College of Health Solutions, Arizona State University, Phoenix, AZ 85004, USA

6 Biodesign Center for Personalized Diagnostics, Biodesign Institute, Arizona State University, Tempe, AZ 85287, USA.

*Correspondence to: Hao.Yan@asu.edu, Yung.Chang@asu.edu 


\section{Supporting information}

\section{RNA-OG sequence:}

5'GGGAGAGAGCUCGAGCGAACACUAGCCACUUGAUCACGCUGAGCGCUCGUACA AUGAAACACAGGUGUGUCAGUGCUAUGCACGUUCGAAGAGCUGUAUCAGCGUUC GUGUGAAUGAGUUCAACGGAGUGUUGACUAAGCCGGUUGCUACAUUUCUGUAGC ACACAUAGUCAAGAUUUGCACCAGACGAUACUCUCCCUCAGUCCUGUUUAUGCAA GUCGUCGUAGUCCUGACGUACUUCCUAAGCUCGUCACUGUACUGAUGAUUCCACU GAUCAAGAUGCACGUAUCUUCAGUUUCCUGAAGAUCGGAGUAGGCACUAUAAUC GACAAGUAACGCUUACGAUUCCAUCACGAGUGACUUACCUGAACCAUAACUGACA AGGGACCACGCAGAGGUCAUACUCACAGGACUUCAAAUCUUGAGUCGGGUUCGA UCAUUUCUGAUCGAGACACCAGUGUGAGGUAAUCGUACGUCACUUGAUAGGAGC UCUAAGUAGAGUUGAGAGCCUGUUAACUAGACACGAGUAACGAGGUUAGCCUGU ACGAGAUAUCGGGCUAUAGUGCGGACACGAUUGCACCAUUUCUGGUGCAACGAA GGUGAGCAUGUAUGGACAGGUCAGUGUGACUCAAGUCGAUAGUCCAAGUAGGUU AUCGACUCGCAUAGCUCAAUGACUGUCAUCGCCAGAGUAUCUAGGUGUCUACCUC ACGAAUCGCGUCGUUACAUUUCUGUAACGCUCAUACCGUGCUGAUCUAUGGGACA CGUCGCUUAUUCUUGGGUCAUGACAGUUGCCACAAACAAGGCACGACCUCACACC UGCGAACUUCAAGCGUUAGGCUGACGUUACAUGCUUGCGUGCACUGAUUCGUUU CCGAAUCAGAGACCUACGAAGCCAGAGUUCGUUCACUAUCAUAAGUGCACUGAUG CAUUUGUGCCAACAUUGAAGGCAUCGAGAUAAACAGCCGUCUUAAUCAAGUGAG CACCUGAGAUCAGCAUGAUUCGUCUAUUUCUAGACGAAUCAACUUCCAUUCAGGU GCCUUGCUACUUAAGACGGGAUUAACUCUCGAUGCAACGUGCAUUGGCACAACUC GUGAUGUGCACUUUCACACUGGAACGAACUCUGGCUUCGUAGGUCUGUUUGUCA UUUCUGACAAACUGCACGCACUGUUAGUACGUCAGCCACUUAACCGAAGUUCGUC AUAAGUAGGUCGUGCGACUACGAUGGCAACUUCUACUUACCAAGAAUAAGCGAC GUGUCCCAUAAUGGAAGUCGGUAUGAGGUAUGACUUUCGUCAUACACGCGAUUC CACAAUGUGACACCUAACGUUUGAGGCGAUGACCUGAUACAAGCUAUGCAUGGU UCAAACCUACUUGGACUAUCGACUUGAGAUGAUAGUACCUGUCCAACUAACAGCA CCUUCGAUACCUCGUUUCCGAGGUAUUCGUGUCCUGUGUCAGGCCCGAUAUUAAU GUGUGGCUAACCCUUAGGAACGUGUCUAGUUAACAGGCUCUCAACGUCAUGACG AGCUCCUAGUAGCAAGCGUACGAUACAUUGUGACUGGUGUCUACUGGAUUUCUC CAGUAACCCGACUCCGACUACAAAGUCCUGACUCAUUCACCUCUGCGUGGUCCCU UGUCAGUUGAGUCGAUGGUAAGUCAAUGCAUCAGGAAUCGUGGUUAAGUCUUGU CGAUCUGACACACUACUCCGCUGUCCUGUUUCCAGGACAGACGUGCAUUAGCAGU UGUGGAAUCAUCAGUACAGUGACGAGUCGUUACUGUACGUCAGCUUGUUUGCGA CUUGCAGUUAAUCGACUGAGGGUCAAACGUGUCUGGUGUGUAGUCGGACUAUGU GACGUUCAUUUCUGAACGUACCGGCUUAGUCAACACUCCGUUGAUGAGUAUGAC ACGAACGAGUCAUUGGCUCUUCGCUUCAAUGUAGCACUGAACUUAUGAUGUUUC AUACACAUUACGCUCAGCGAACUGCUAUGGCUAGUGUUCG3'

DNA template construction: 
Two DNA fragments were synthesized by Biobasic Inc. company and cloned into the pUC57 vector. The sequence information of these two DNA fragments are as follows:

DNA fragment 1, HindIII+XbaI:

5'AAGCTTAATACGACTCACTATAGGGAGAGAGCTCGAGCGAACACTAGCCACTTGA TCACGCTGAGCGCTCGTACAATGAAACACAGGTGTGTCAGTGCTATGCACGTTCGAA GAGCTGTATCAGCGTTCGTGTGAATGAGTTCAACGGAGTGTTGACTAAGCCGGTTGC TACATTTCTGTAGCACACATAGTCAAGATTTGCACCAGACGATACTCTCCCTCAGTC CTGTTTATGCAAGTCGTCGTAGTCCTGACGTACTTCCTAAGCTCGTCACTGTACTGAT GATTCCACTGATCAAGATGCACGTATCTTCAGTTTCCTGAAGATCGGAGTAGGCACT ATAATCGACAAGTAACGCTTACGATTCCATCACGAGTGACTTACCTGAACCATAACT GACAAGGGACCACGCAGAGGTCATACTCACAGGACTTCAAATCTTGAGTCGGGTTC GATCATTTCTGATCGAGACACCAGTGTGAGGTAATCGTACGTCACTTGATAGGAGCT CTAAGTAGAGTTGAGAGCCTGTTAACTAGACACGAGTAACGAGGTTAGCCTGTACG AGATATCGGGCTATAGTGCGGACACGATTGCACCATTTCTGGTGCAACGAAGGTGA GCATGTATGGACAGGTCAGTGTGACTCAAGTCGATAGTCCAAGTAGGTTATCGACTC GCATAGCTCAATGACTGTCATCGCCAGAGTATCTAGGTGTCTACCTCACGAATCGCG TCGTTACATTTCTGTAACGCTCATACCGTGCTGATCTATGGGACACGTCGCTTATTCT TGGGTCATGACAGTTGCCACAAACAAGGCACGACCTCACACCTGCGAACTTCAAGC GTTAGGCTGACGTTACATGCTTGCGTGCACTGATTCGTTTCCGAATCAGAGACCTAC GAAGCCAGAGTTCGTTCACTATCATAAGTGCACTGATGCATTTGTGCCAACATTGAA GGCATCGAGATAAACAGCCGTCTTAATCAAGTGAGCACCTGAGATCAGCATGATTC GTCTATTTCTAGA3'

DNA fragment 2, XbaI + EcoRI:

5'TCTAGACGAATCAACTTCCATTCAGGTGCCTTGCTACTTAAGACGGGATTAACTCT CGATGCAACGTGCATTGGCACAACTCGTGATGTGCACTTTCACACTGGAACGAACTC TGGCTTCGTAGGTCTGTTTGTCATTTCTGACAAACTGCACGCACTGTTAGTACGTCAG CCACTTAACCGAAGTTCGTCATAAGTAGGTCGTGCGACTACGATGGCAACTTCTACT TACCAAGAATAAGCGACGTGTCCCATAATGGAAGTCGGTATGAGGTATGACTTTCGT CATACACGCGATTCCACAATGTGACACCTAACGTTTGAGGCGATGACCTGATACAAG CTATGCATGGTTCAAACCTACTTGGACTATCGACTTGAGATGATAGTACCTGTCCAA 
CTAACAGCACCTTCGATACCTCGTTTCCGAGGTATTCGTGTCCTGTGTCAGGCCCGAT ATTAATGTGTGGCTAACCCTTAGGAACGTGTCTAGTTAACAGGCTCTCAACGTCATG ACGAGCTCCTAGTAGCAAGCGTACGATACATTGTGACTGGTGTCTACTGGATTTCTC CAGTAACCCGACTCCGACTACAAAGTCCTGACTCATTCACCTCTGCGTGGTCCCTTG TCAGTTGAGTCGATGGTAAGTCAATGCATCAGGAATCGTGGTTAAGTCTTGTCGATC TGACACACTACTCCGCTGTCCTGTTTCCAGGACAGACGTGCATTAGCAGTTGTGGAA TCATCAGTACAGTGACGAGTCGTTACTGTACGTCAGCTTGTTTGCGACTTGCAGTTA ATCGACTGAGGGTCAAACGTGTCTGGTGTGTAGTCGGACTATGTGACGTTCATTTCT GAACGTACCGGCTTAGTCAACACTCCGTTGATGAGTATGACACGAACGAGTCATTGG CTCTTCGCTTCAATGTAGCACTGAACTTATGATGTTTCATACACATTACGCTCAGCGA ACTGCTATGGCTAGTGTTCGAATTC3'

The two DNA fragments were then cleaved from the plasmids with HindIII+XbaI and XbaI+EcoRI respectively, and subcloned into HindIII+EcoRI linearized pUC19 vector. The plasmid was linearized with EcoRI for in vitro transcription reactions.

\section{Construction of Tfr-OVA gene into pMuLE Lenti Dest iRFP vector:}

The plasmid pcDNA3-TfR-OVA containing the TfR-OVA gene was obtained from addgene (Plasmid \#64600). The TfR-OVA gene, flanked by Hind III and Xba I restriction sites, was digested and cloned into the same sites of pMuLE ENTR SV40 L5-L2 (Plasmid \#62095) entry vector downstream of the SV40 promoter. Since this pMuLE ENTR SV40 L5-L2 vector contained attB5 and attL2 sites instead of attL1 and attL2 sites required for gateway cloning, an attL1 site was introduced by replacing the attB5 region using the one-step multiple-site mutagenesis method with the following forward and reverse primers: Forward 5'CTTTTTTATAATGCCAACTTTGTACAAAAAAGCAGGCTTATACAAAAGTTGCTGTGG AATGTG-3' and Reverse 5' AGCCTGCTTTTTTGTACAAAGTTGGCATTATAAAAAAGCATTGCTCATCAATTTGTTG CAACG-3'. The PCR conditions used with Pfu Turbo DNA polymerase were as follows: Predenaturation- $95^{\circ} \mathrm{C}$ for $5 \mathrm{mins}, 20$ cycles of denaturation $-95^{\circ} \mathrm{C}$ for $30 \mathrm{sec}$, Annealing $-55^{\circ} \mathrm{C}$ for $30 \mathrm{sec}$, Extension $-72^{\circ} \mathrm{C}$ for 6 mins followed by final extension at $72^{\circ} \mathrm{C}$ for 30 mins. The purified PCR product was transformed into the E.coli DH5 $\alpha$ strain and confirmed by sequencing. The expression vector was prepared using the entry vector pMuLE ENTR SV40 containing TfR- 
OVA gene and the destination vector pMuLE Lenti Dest iRFP (Plasmid \#62176) that can coexpress iRFP under the control of a PGK promotor by gateway cloning. The cloning reaction used was as follows: $50 \mathrm{ng}$ of entry clone and $150 \mathrm{ng}$ of destination vector were incubated at $25^{\circ} \mathrm{C}$ with LR Clonase II enzyme in TE buffer, $\mathrm{pH} 8.0$ for 1 hour and the reaction was stopped using Proteinase $\mathrm{K}$ solution before transforming into E.coli $\mathrm{DH} 5 \alpha$. The final expression vector containing both TfR-OVA under the SV40 promotor and iRFP under the PGK promotor were confirmed by sequencing.

\section{Lentivirus based transduction of TfR-OVA gene:}

The lentiviral cloning vector pMuLE Lenti Dest iRFP containing the TfR-OVA gene was prepared using Qiagen's mini prep kit and the purity was verified. Lentiviral vector was produced using LENTI-Smart kit with its standard small scale production protocol. $3 \mu \mathrm{g}$ of TfROVA lentiviral cloning vector was added to $100 \mu 1$ of re-suspended LENTI-Smart solution and incubated for 15 minutes at RT. This LENTI-Smart plasmid solution was added to $1 \times 10^{6} \mathrm{HEK}$ $293 \mathrm{~T}$ cells in a 6-well plate containing $2 \mathrm{ml}$ of pre-warmed DMEM growth medium and incubated at $37^{\circ} \mathrm{C}$ with $5 \% \mathrm{CO}_{2}$. The culture medium was replaced with fresh medium after 12 hours and the lentiviral vector titer was harvested after 36 hours and 48 hours. The pooled supernatants were centrifuged at $2000 \mathrm{rpm}$ and filtered using $0.45 \mu \mathrm{m}$ PVDF filters to remove any viral or cell debris. Further, lentiviral particles were concentrated using Amican Ultra 100 $\mathrm{kDa}$ filter and stored in aliquots at $-80^{\circ} \mathrm{C}$ until used. A CT- 26 cell line obtained from ATCC was transduced by adding TfR-OVA expressing lentiviral vector and incubated overnight at $37^{\circ} \mathrm{C}$ with $5 \% \mathrm{CO}_{2}$. Culture medium was exchanged with fresh medium and incubated further to study the transgene expression. 


\section{Supplementary figures:}

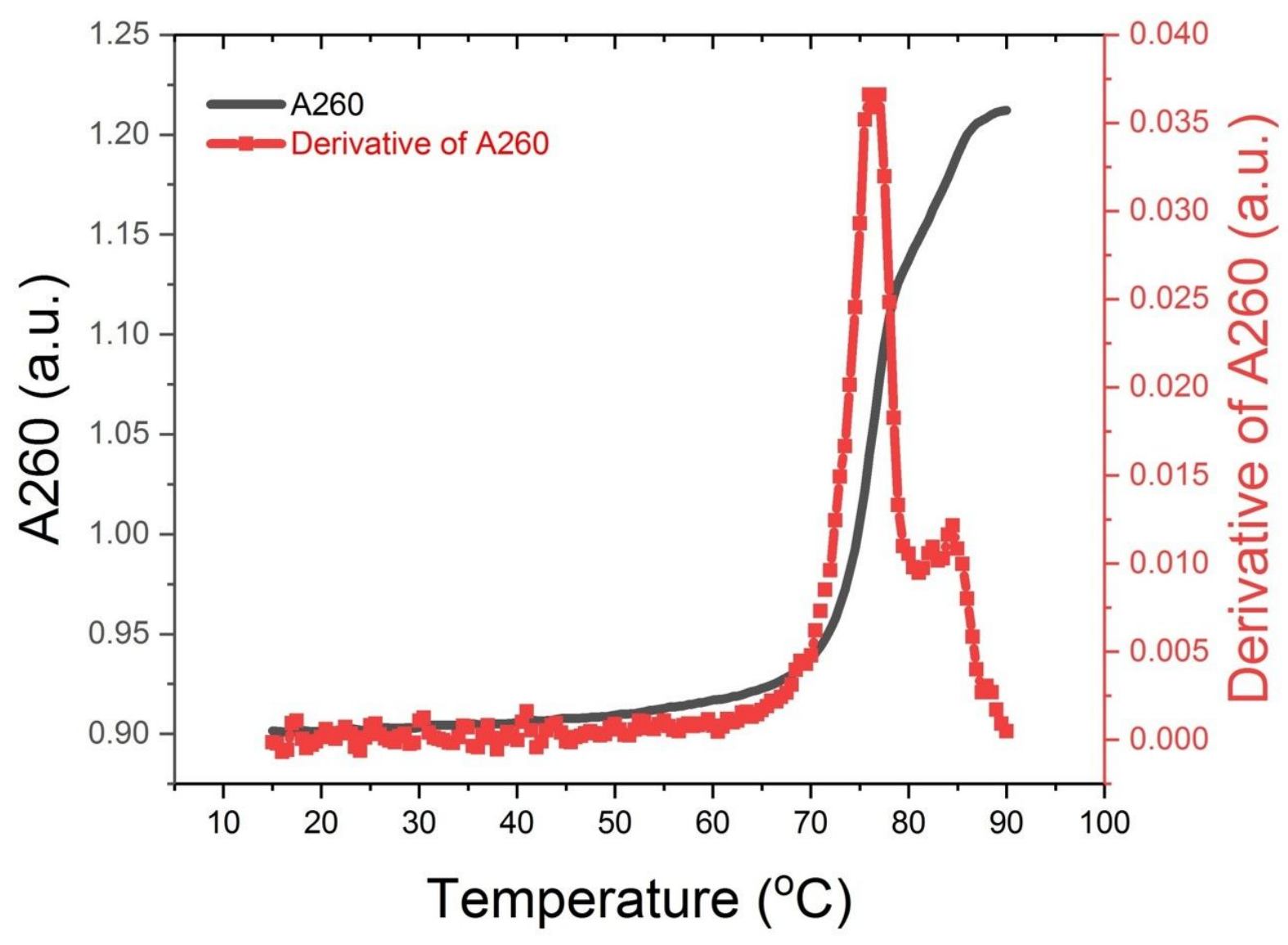

Supplementary figure S1: UV melting curve of the RNA-OG. The UV absorbance of RNA at $260 \mathrm{~nm}$ (A260) was plotted as a function of temperature (black). Two melting transitions were observed by taking the first derivative of A260 vs. temperature (red). The tall and sharp transition $\left(\sim 76^{\circ} \mathrm{C}\right)$ corresponds to the melting of paranemic cohesion; and the short transition $\left(\sim 84^{\circ} \mathrm{C}\right)$ corresponds to the melting of the remaining hybridized regions. 


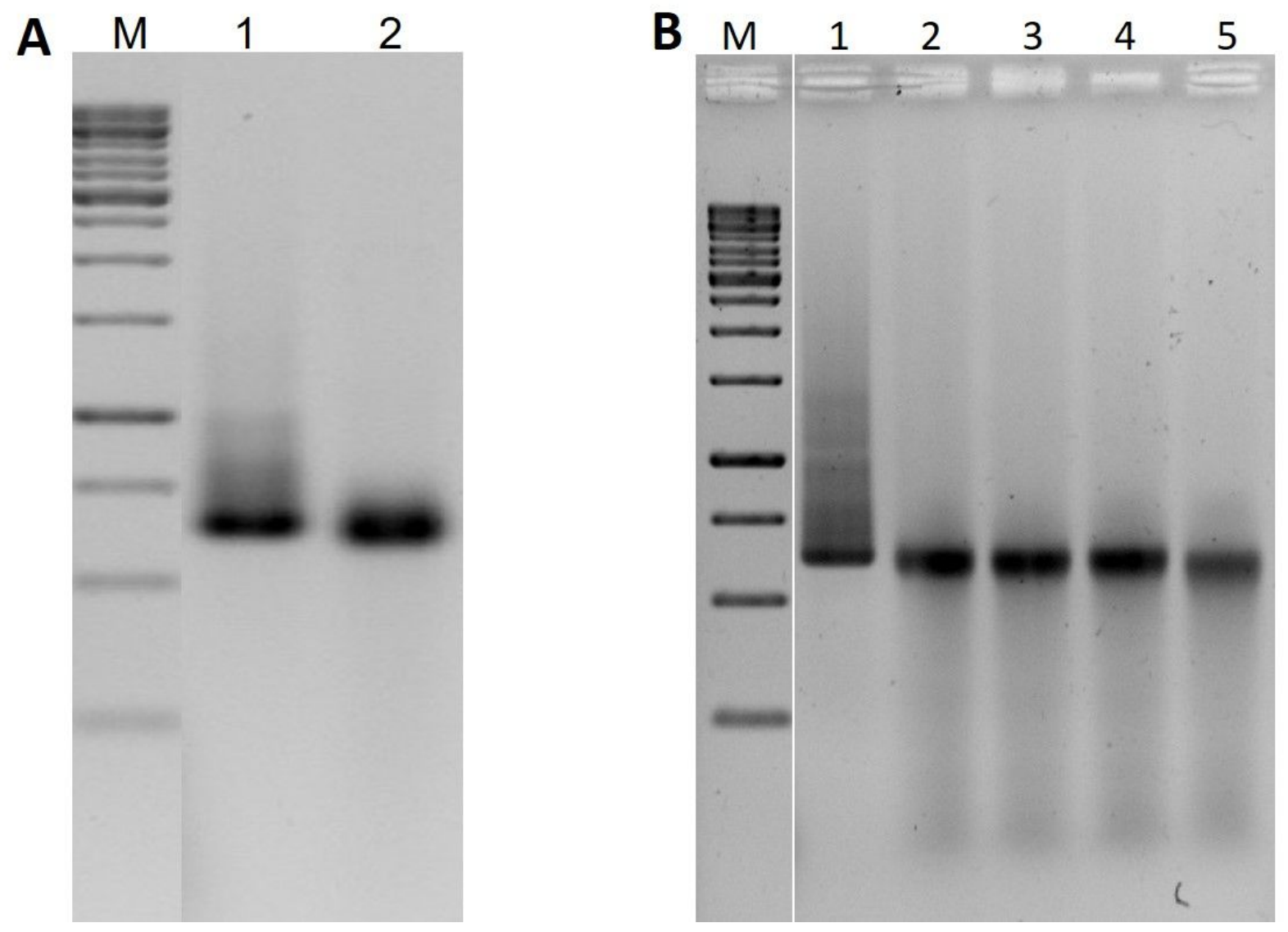

Supplementary figure S2: Stability evaluation of RNA-OG. A. RNase I digestion of RNAOG. $1 \mu \mathrm{g}$ of RNA-OG (lane 1) was incubated with $1 \mathrm{U}$ of RNase I at room temperature for 20 minutes (lane 2). Unit definition: One unit of the RNase I enzyme catalyzes degradation of 100 ng of E. coli rRNA per second into acid-soluble nucleotides at $37^{\circ} \mathrm{C}$. B. RNA-OG stability in $50 \%$ mouse serum. $1 \mu \mathrm{g}$ of RNA-OG (lane 1 ) was incubated with $50 \%$ mouse serum at $37^{\circ} \mathrm{C}$ for 1 hour (lane 2), 2 hours (lane 3), 4 hours (lane 4) and 20 hours (lane 5). M denotes $1 \mathrm{~kb}$ DNA marker. 
RNA-OG

24hrs

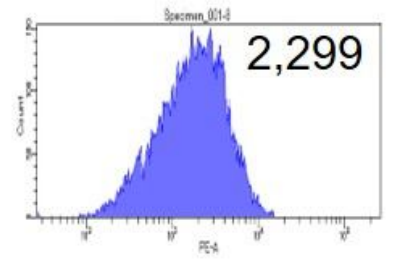

2hrs
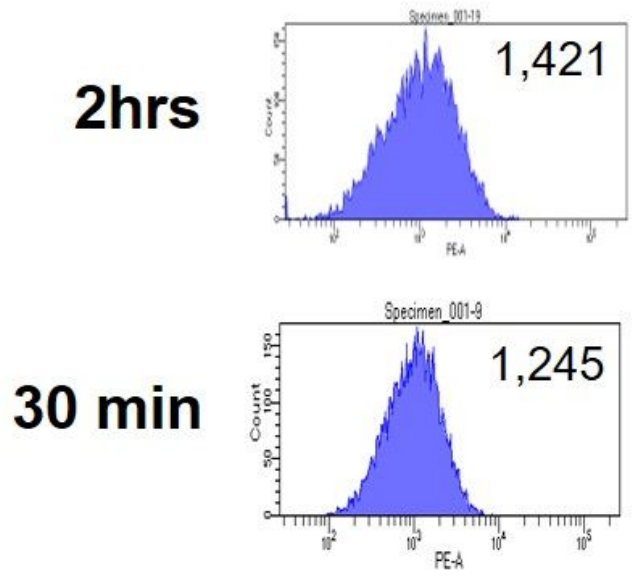

PolyIC
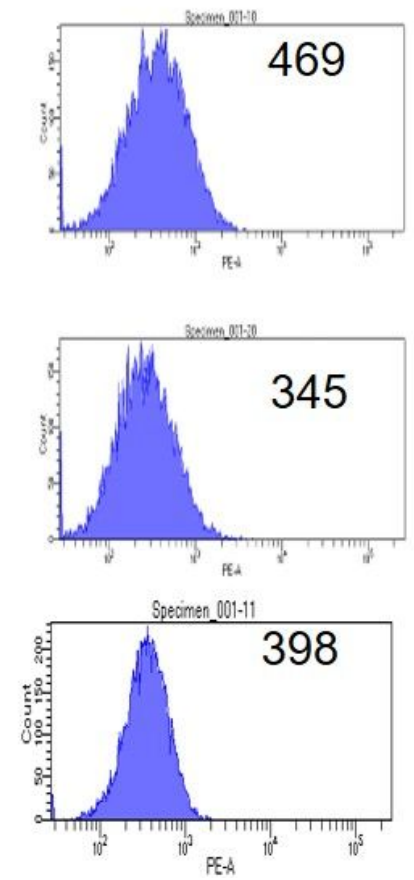

\section{PBS}

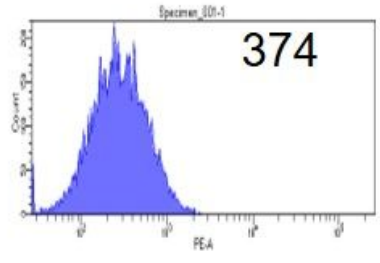

Supplementary figure S3: Rapid stimulation of RAW 264.7 cells with RNA-OG. Murine macrophage cell line, RAW 264.7 cells, were incubated with $5 \mu \mathrm{g}$ of RNA-OG or PolyIC at $37^{\circ} \mathrm{C}$ for various time points. The cells were then stained with PE labeled anti-CD40 antibody and analyzed by FACS. The MFI numbers are shown. 


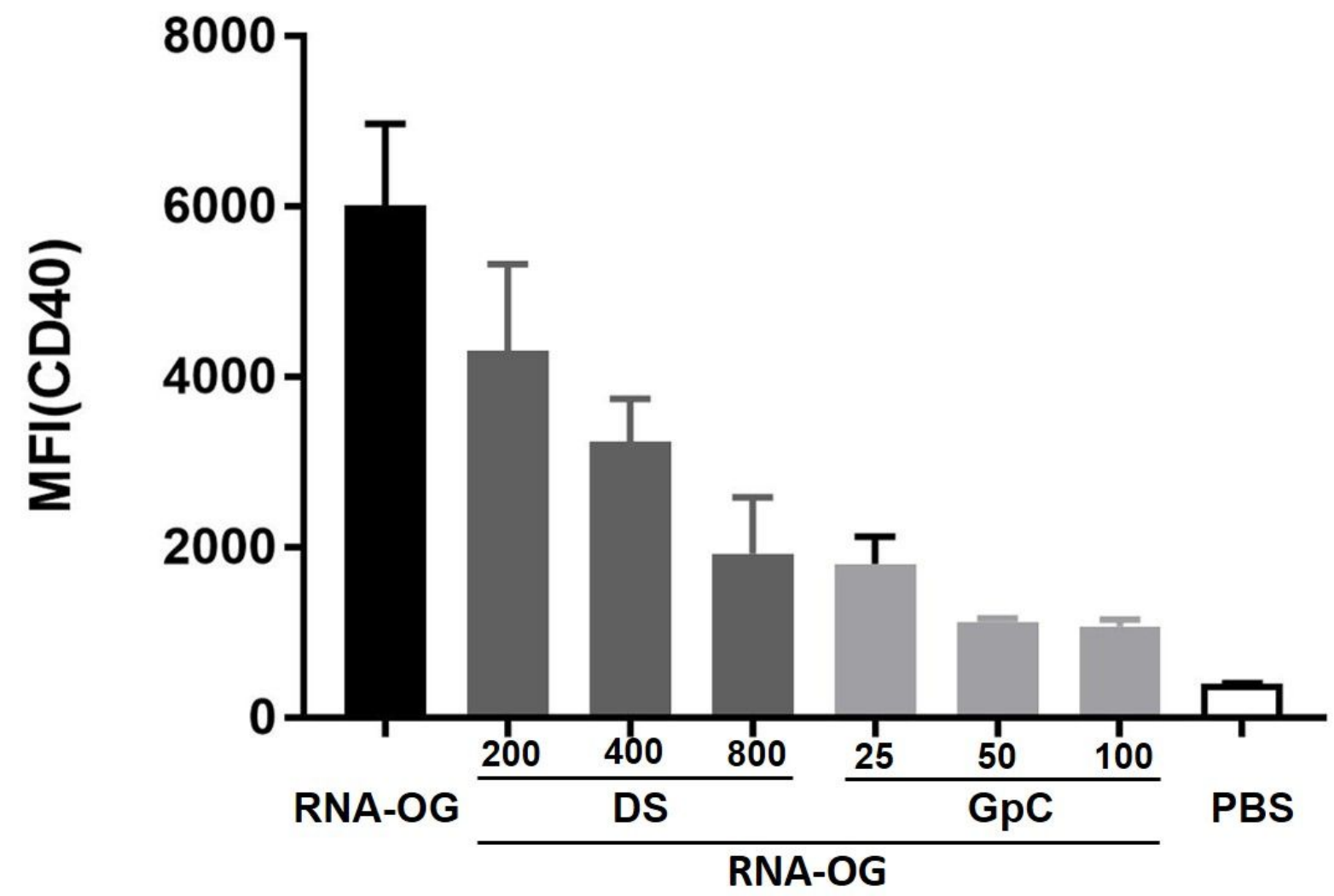

Supplementary figure S4: Inhibition of RNA-OG mediated macrophage activation (CD40) by Dextran sulfate (DS) and GpC. RAW 264.7 cells were pre-incubated with DS or GpC at various concentrations for 30 minutes at $37^{\circ} \mathrm{C}$. The numbers listed on top of $\mathrm{DS}$ or $\mathrm{GpC}$ are the inhibitor concentrations utilized $(\mu \mathrm{g} / \mathrm{ml})$. The RNA-OG $(5 \mu \mathrm{g} / \mathrm{ml})$ was added as a stimulator for an additional 60 minutes. The cells were stained with PE-labeled anti-CD40 antibody and analyzed by FACS. The CD40 MFI numbers were shown. 
A

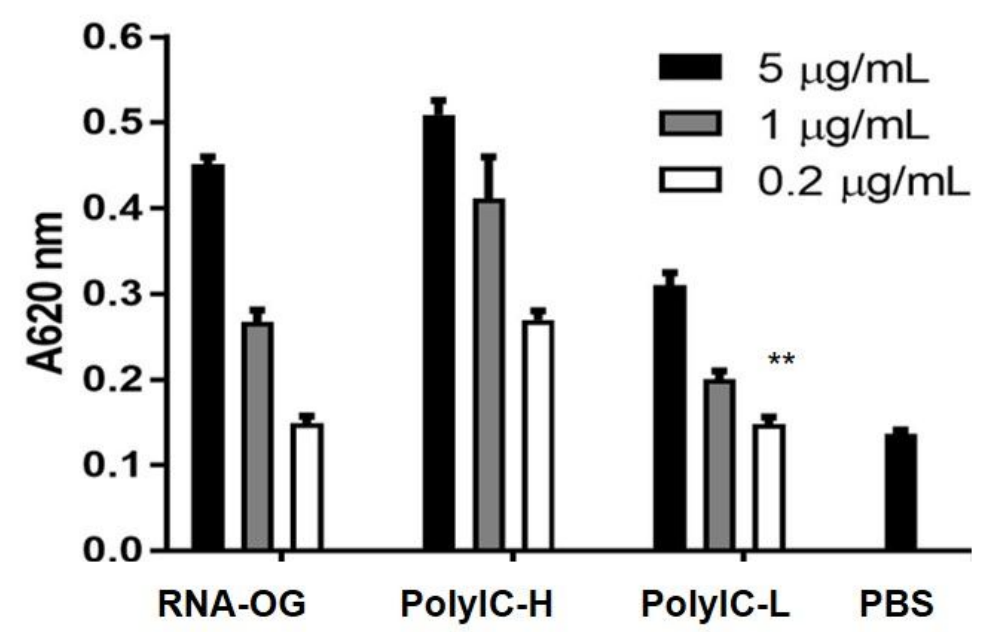

B

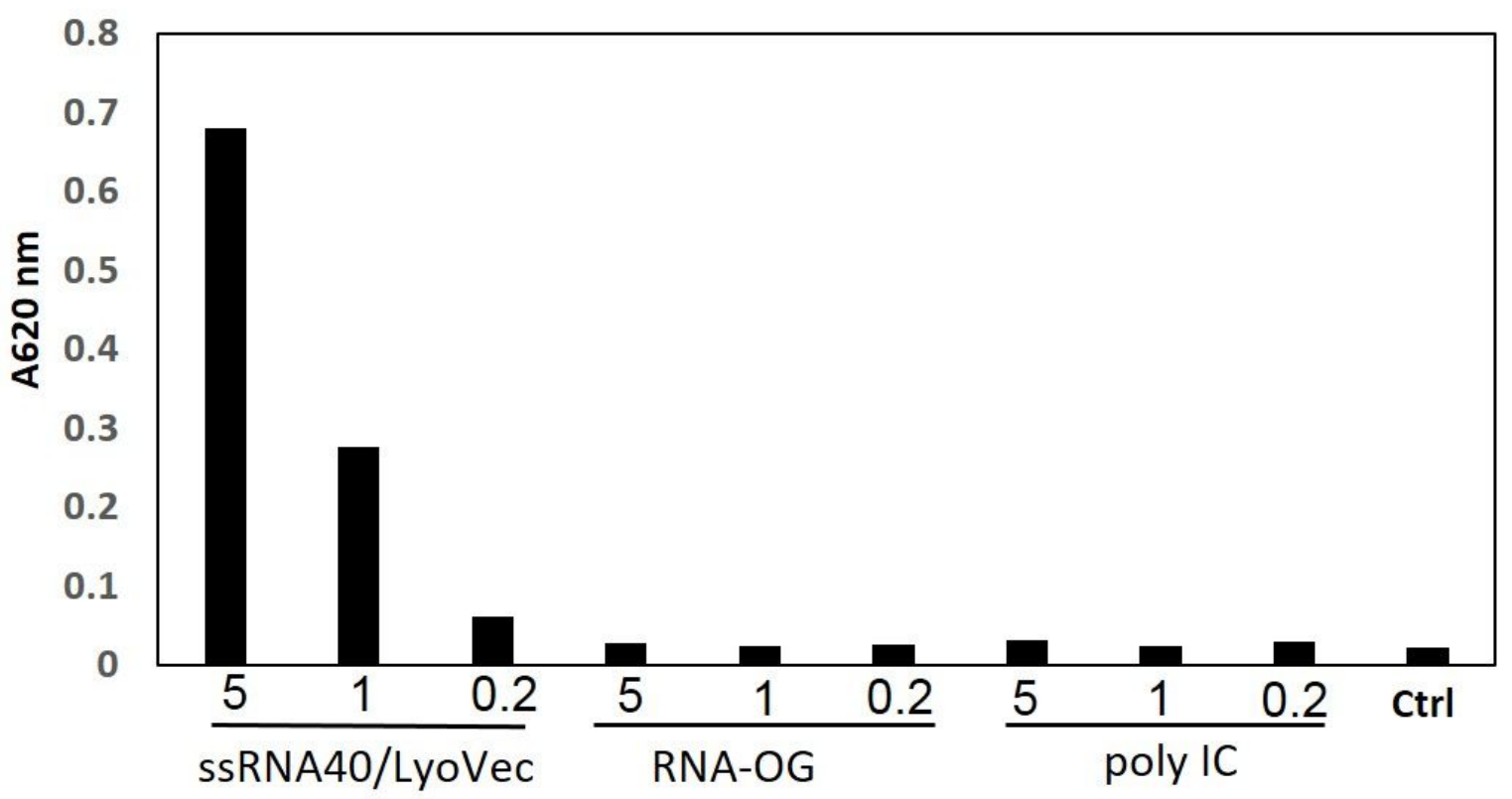

Supplementary figure S5: Induction of TLR3-dependent activation. A. In vitro stimulation of a NF-KB-based HEK-297-TLR3 reporter cell line, HEK-Blue mTLR3, with RNA-OG, PolyIC-H, and PolyIC-L at different concentrations for 20 hours; ** indicates $\mathrm{p}<0.01$ between PBS and other groups treated with $5 \mu \mathrm{g} / \mathrm{ml}$ RNA samples. B. In vitro stimulation of a TLR7 reporter cell line, HEK-Blue mTLR7, in which ssRNA40 is a short U-rich ssRNA, which is a mTLR7/hTLR8 agonist. 


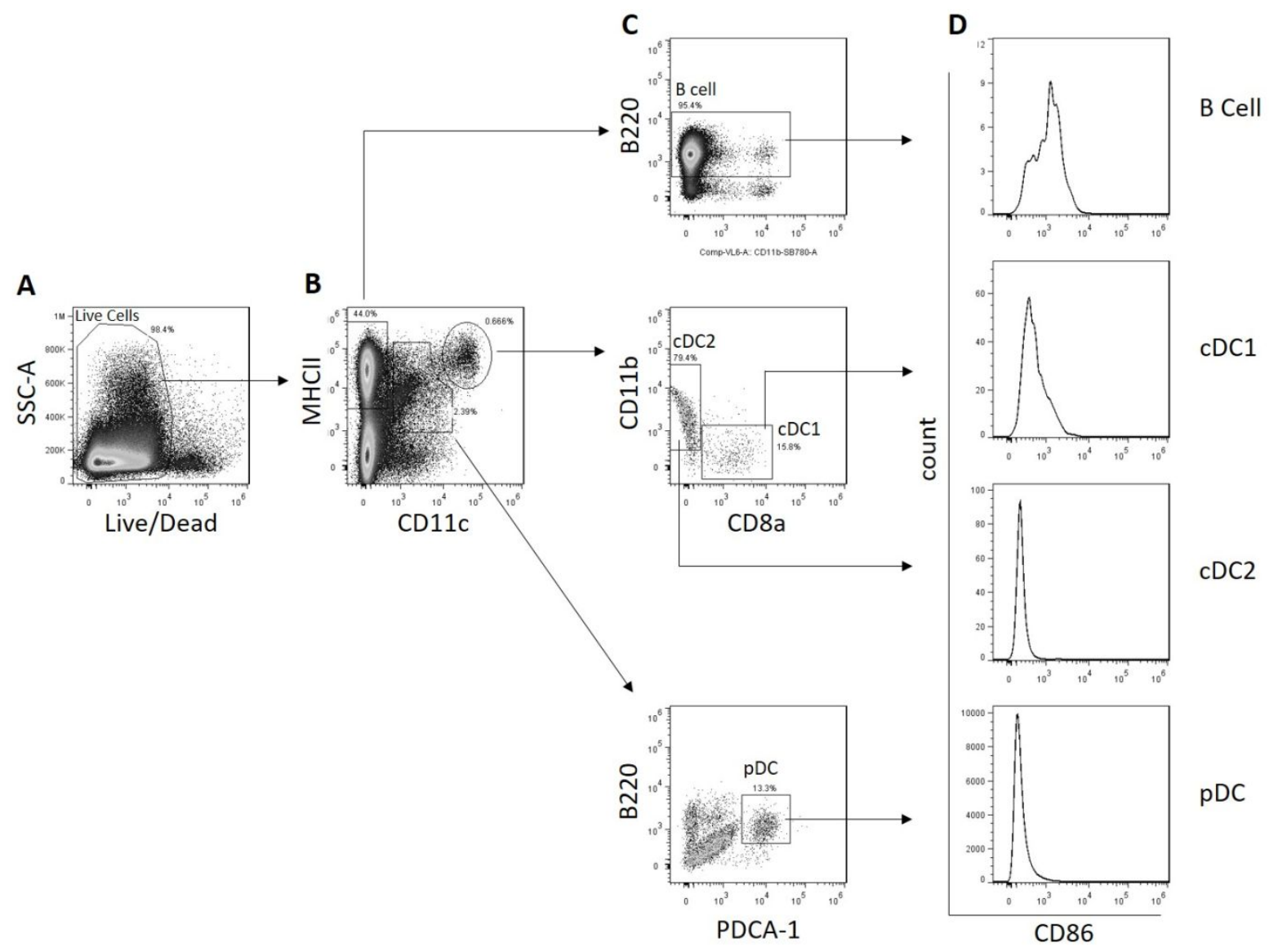

Supplementary figure S6: Strategy for gating APCs. Murine splenocytes, after RBC lysis, were stained with a panel of fluorophore conjugated antibodies and various populations of antigen presenting cells (APCs) were analyzed and displayed by flow cytometry. A. Live cells, gated by Ghost Red 780 viability dye, were displayed with CD11c and MHCII (B) to identify APCs by their MHCII profile, majority of which were positive. C. B cells were identified as CD11c-MHCII+B220+CD11b+/-. Dendritic cells (DCs) were separated into conventional DC 1 (cDC1) (MHCII(high)CD11c+B220-CD8a+), conventional DC 2 (cDC2) (CD11c+B220-CD8a$\mathrm{CD} 11 \mathrm{~b}+$ ), and plasmacytoid DCs (pDCs) (MHCII(low)CD11c+B220+PDCA1+). D. The stimulation of each APC was then measured by their mean fluorescent intensity (MFI) of CD86. 

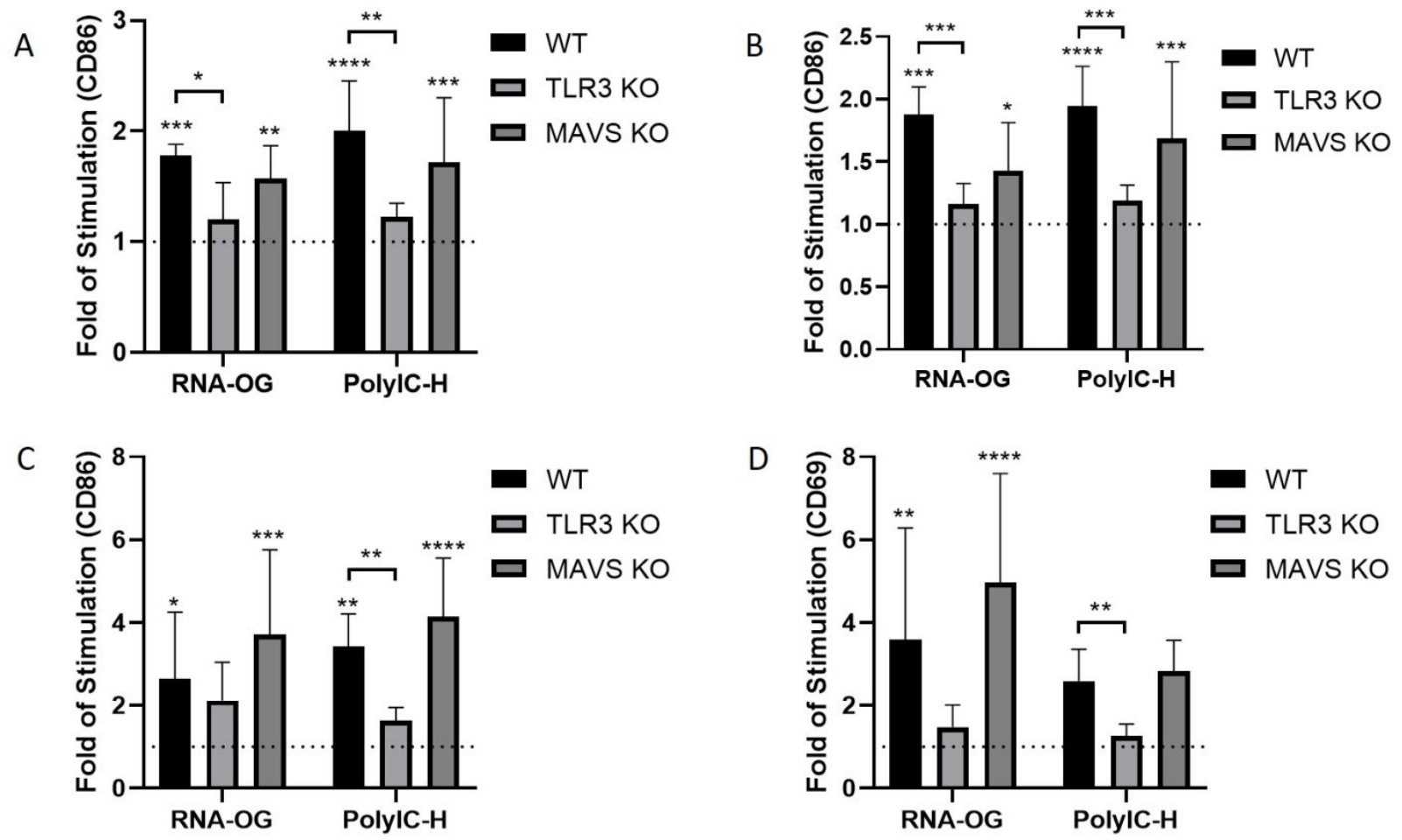

Supplementary figure S7: Activation of dendritic, B, and NK cells induced by RNA-OG and PolyIC-H in WT, TLR3 KO, and MAVS KO mice. Splenocytes from TLR3 knockout, MAVS knockout, and wild type mice (WT) were stimulated with $5 \mu \mathrm{g} / \mathrm{mL}$ RNA-OG or PolyIC-H for 24 hours. Activation was measured by flow cytometry and displayed as folds of increase in CD86 or CD69 MFI above background for A. conventional DCs (MHCII-high/CD11c+), B. conventional DC2s (MHCII-high/CD11c+/CD8a-/CD11b+), C. B cells (MHCII+/CD11c/B220+), and D. NK cells (B220-CD4-CD8-CD49b+). Stimulation profiles for each set of cells are displayed as mean folds of stimulation $\pm \mathrm{SD}(\mathrm{n}=5-6)$. The PBS group is presented as a dotted line. Paired t-test was used to compare RNA-OG and PBS or compare PolyIC-H and PBS, as well as between strains of mice, where ${ }^{* * * *}, * * *, * *$, and $*$ indicate $\mathrm{p}<0.0001, \mathrm{p}<0.001$, $\mathrm{p}<0.01$, and $<0.05$, respectively. 


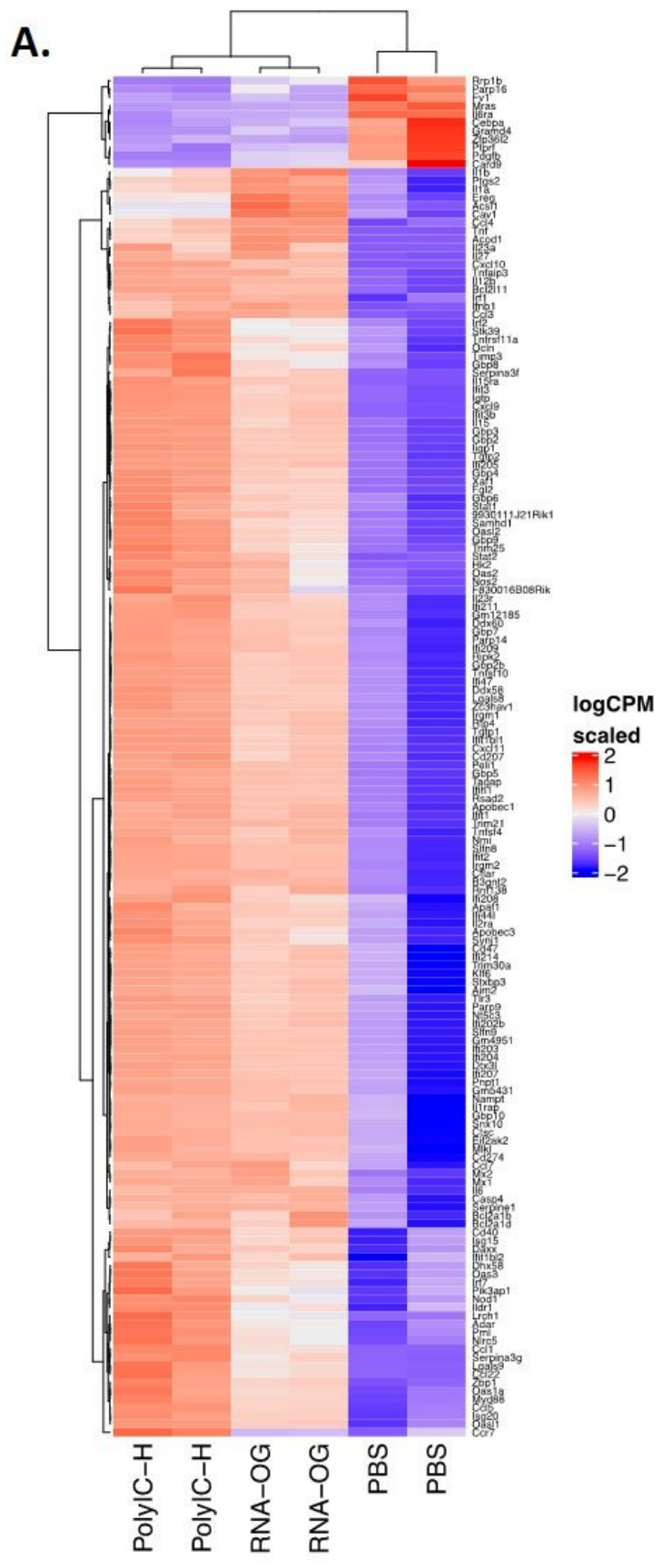

B.

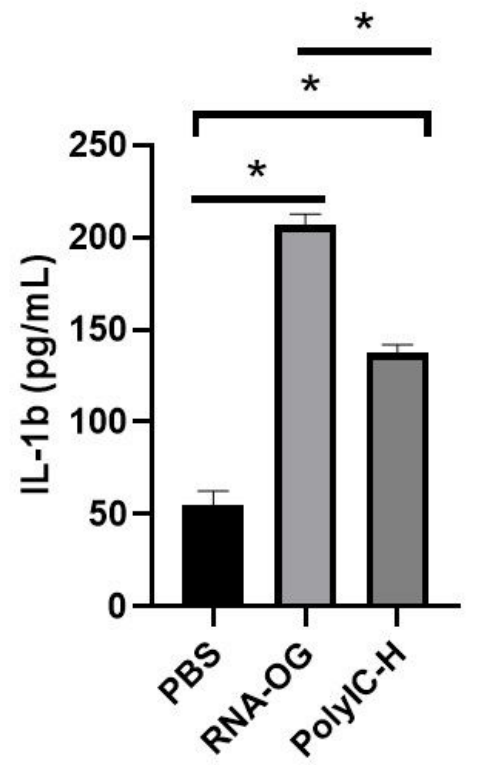

Supplementary Figure S8. RNAseq and cytokine analyses of CD11c+ BMDCs treated with RNA-OGs or PolyIC-H. A. The heat map represents 160 genes that are differentially expressed between PolyIC-H and PBS control or between RNA-OG and PBS control (false discovery rate $<0.1$ and fold change $>1.5$ ) and involved in innate immune responses. Genes are hierarchically clustered based on correlations of z-transformed $\log C P M$ values with replicates for each treatment. Color gradients represent expression levels from high (red) to low (blue). Gene expression levels and statistic scores are available in Supplementary Table 1. B. ILlb levels in supernatant of BMDC cultures stimulated for 24 hours with RNA-OG or PolyIC-H, measured via a multiplex cytokine assay. * indicates $\mathrm{p}<0.05$ between the indicated groups. 

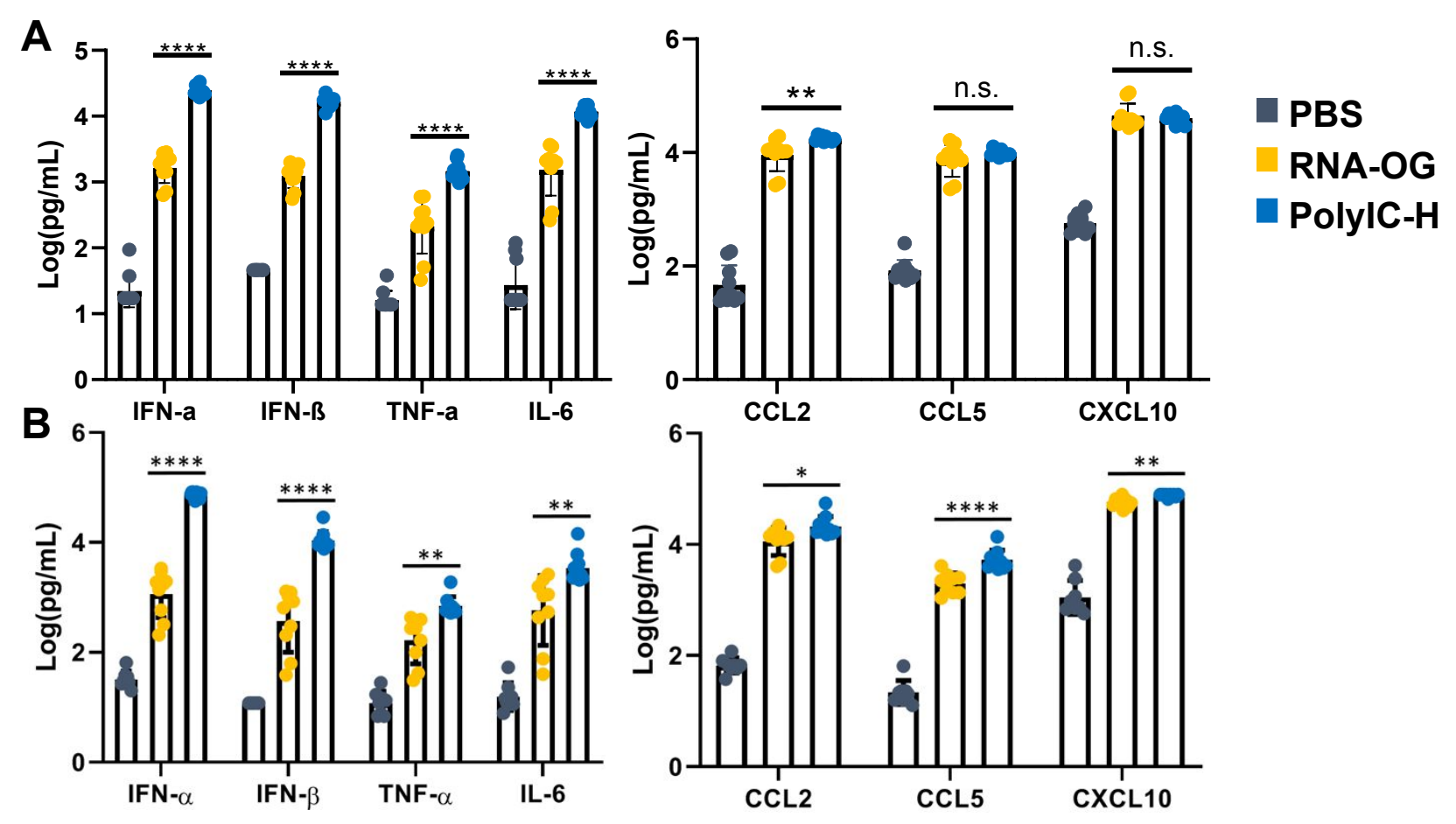

Supplementary figure S9. Cytokine levels in mice administered a large dose of RNA-OG or polyIC. A. Levels of cytokines detected in C57BL6 mice 3 hours post the injection of $250 \mu \mathrm{g}$ RNA-OG or PolyIC-H. B. Levels of cytokines detected in CD1 mice $3 \mathrm{hrs}$ post the injection of $100 \mu \mathrm{g}$ RNA-OG or PolyIC-H. The levels of various cytokines are presented as log-transformed $\mathrm{pg} / \mathrm{ml}$, in which data derived from 4-5 mice per experimental group are displayed as mean \pm SD. The comparison was made between RNA-OG and polyIC. ****,**, and *, indicate $\mathrm{p}<0.0001, \mathrm{p}<0.01$, and $\mathrm{p}<0.05$, respectively. 


\section{A}
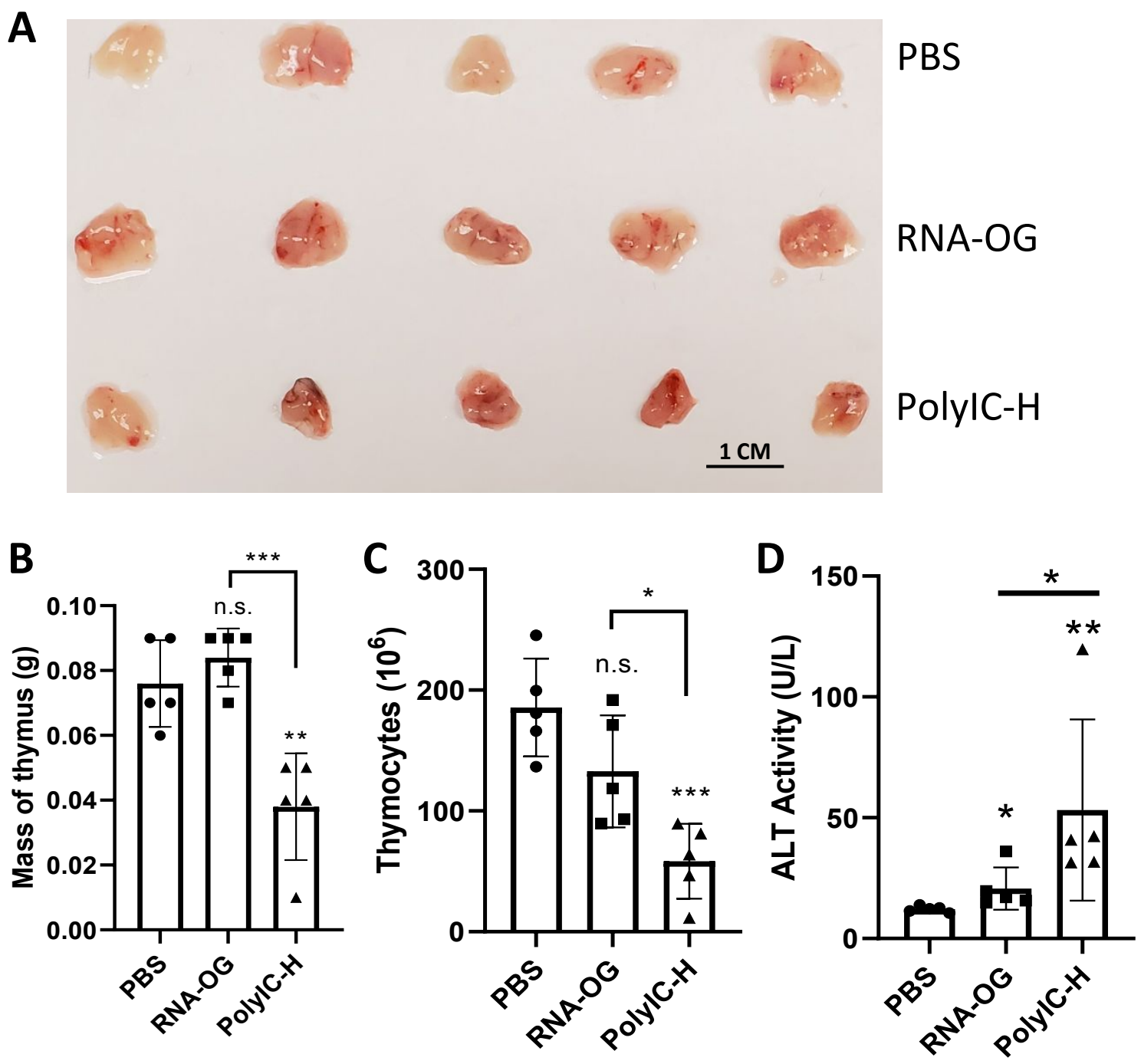

Supplementary figure S10. Thymic involution induced by PolyIC-H, but not RNA-OG. A. Visual comparison of thymi from different treatment groups. B. Masses of thymi from different treatment groups. C. Total cell count of thymocytes retrieved from treated mice. D. Alanine aminotransferase (ALT) activity levels in serum collected $24 \mathrm{hrs}$ post the treatment. Comparisons were made between the nucleic acid compounds and PBS as well as between RNA-OG and PIC. $* * *, * *$, and $*$, indicate $\mathrm{p}<0.001, \mathrm{p}<0.01$, and $\mathrm{p}<0.05$, respectively. 
CT26-OVA-iRFP MTT assay

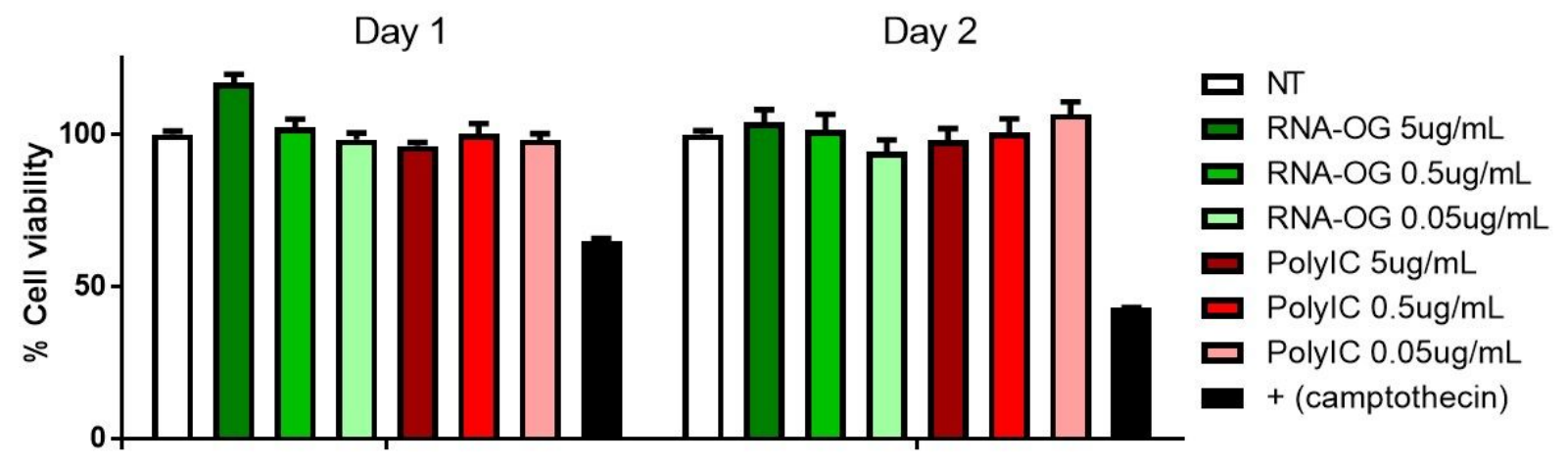

Supplementary figure S11: CT26-OVA-iRFP MTT assay with different doses of RNA-OG or PolyIC. Different doses of RNA-OG or PolyIC were incubated with the CT26-OVA-iRFP cells. The cell viability was evaluated through MTT assay. 


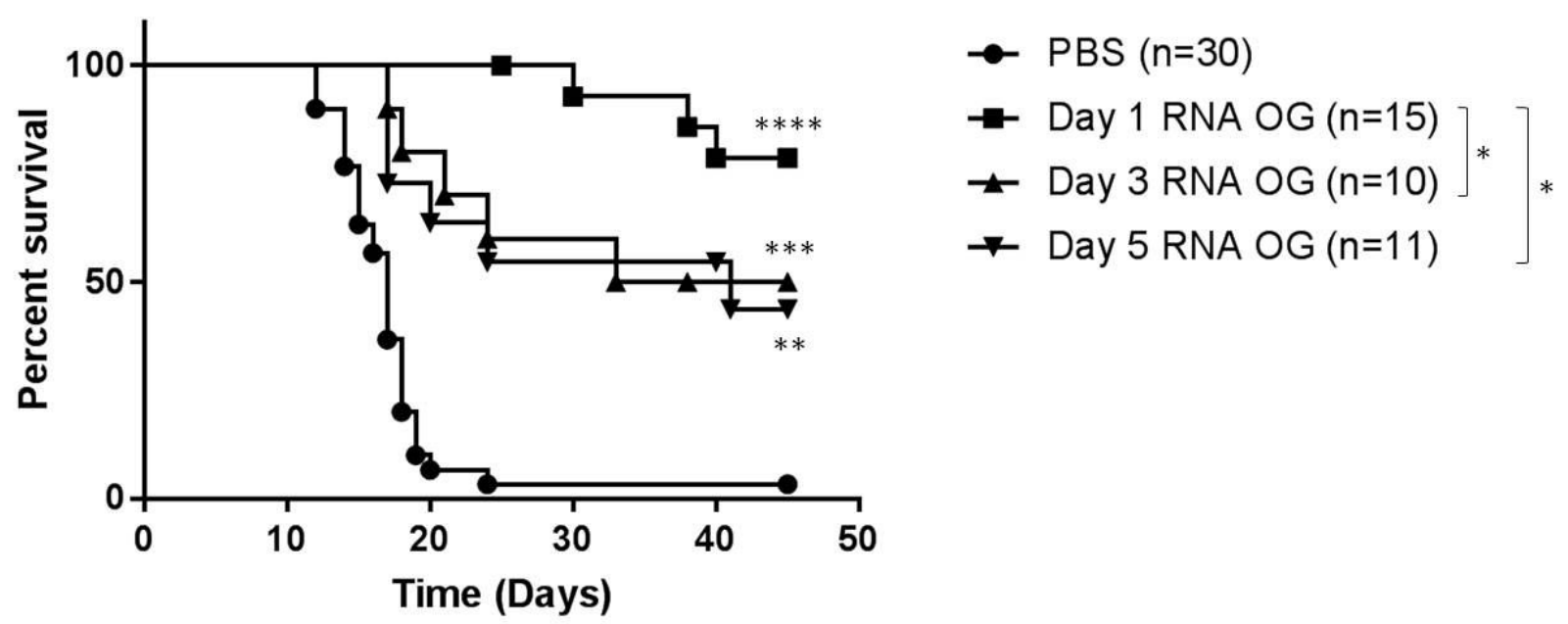

Supplemental figure S12: Anti-tumor effect of RNA-OG. Kaplan-Meier survival curve displaying mice from multiple independent experiments. Mice from three independent experiments received $5 \times 10^{5}$ CT26-iRFP cells via IP injection. Mice began receiving 4 biweekly IP treatments of $100 \mu \mathrm{L}$ of PBS or $16 \mu \mathrm{g}$ of RNA-OG in $100 \mu \mathrm{L}$ PBS on day 1,3 , or 5 for RNAOG and day 1 for PBS. Tumor progression was monitored via the fluorescent intensity of iRFP. Comparisons between PBS and RNA-OG treatments via Gehan-Breslow-Wilcoxon test are shown on the graph. Comparisons between day 1 RNA-OG and days 3 or 5 RNA-OG via GehanBreslow-Wilcoxon test are shown by the legend. $* * * *, * *, * *$, and $*$, indicate $\mathrm{p}<0.0001$, $\mathrm{p}<0.001, \mathrm{p}<0.01$, and $\mathrm{p}<0.05$, respectively. 

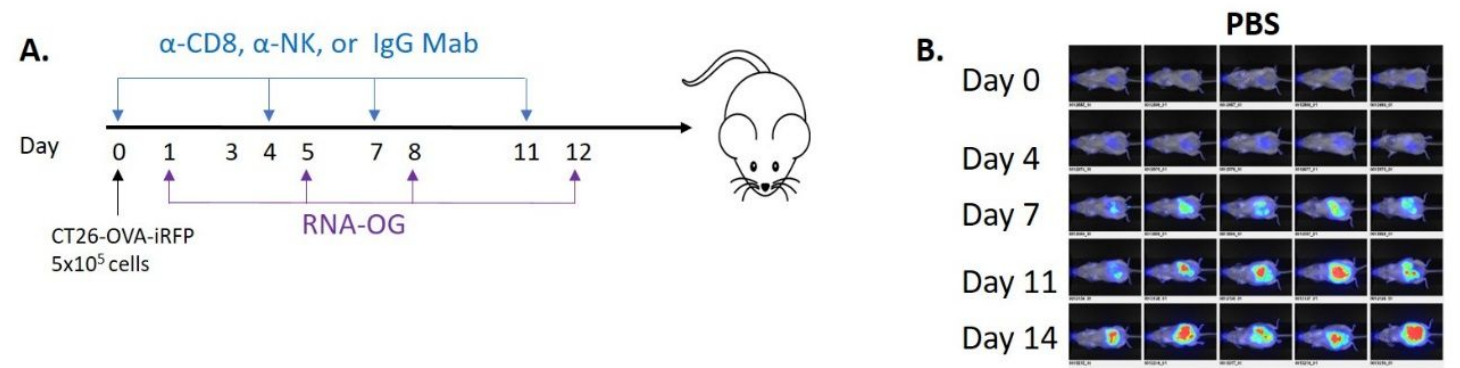

c.

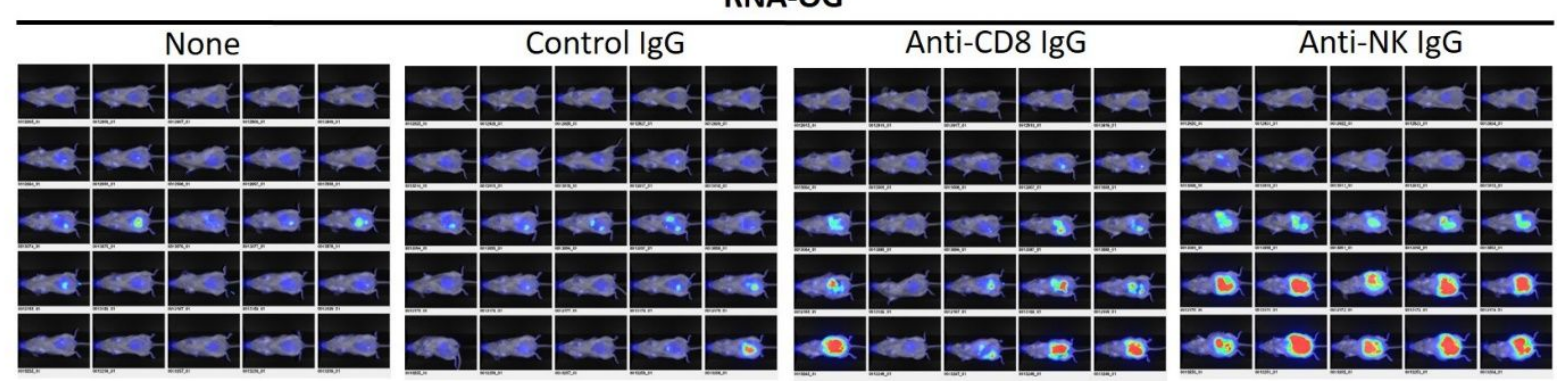

Supplementary Figure S13. Lack of anti-tumor immunity in RNA-OG treated mice that were depleted of CD8 and NK cells. A. A schematic to show treatment schedules in various groups. In vivo depletion of CD8 or NK cells was achieved by injecting monoclonal antibodies (Mab) specific to CD8 or NK cells, respectively. The antibodies were injected on the same day of, but 4 hours post tumor injection. RNA-OG was administered one day post the antibody treatment $(100 \mu \mathrm{g} /$ dose for total four doses). An irrelevant IgG was included as a negative control for CD8/NK depletion. B. Tumor growth in the PBS control mice. C. Tumor growth in mice treated with RNA-OG with or without targeted depletion of CD8 or NK cells. 
A.

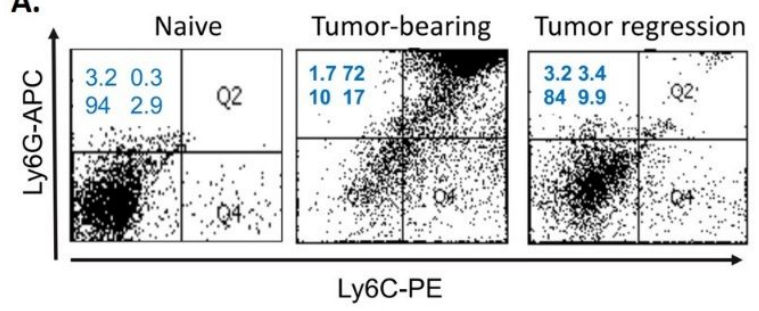

Ly6C-PE

C. T cell Proliferation

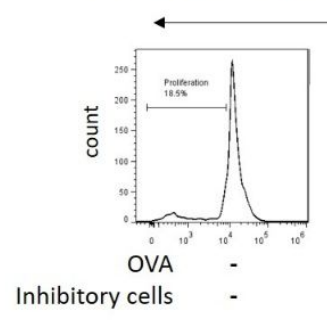

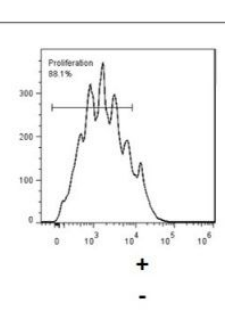

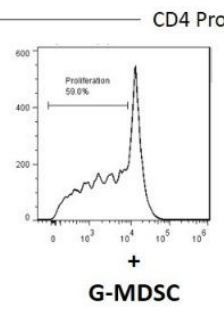

B. MDSC gating

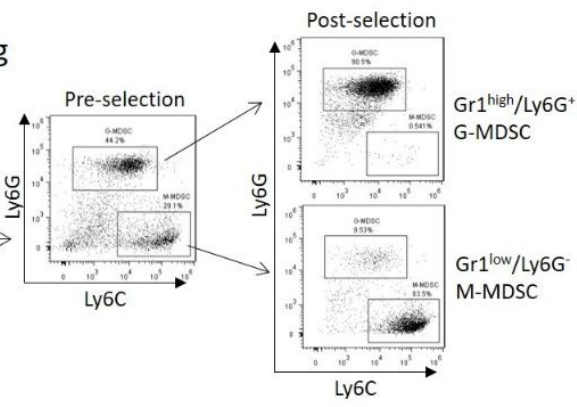

D.

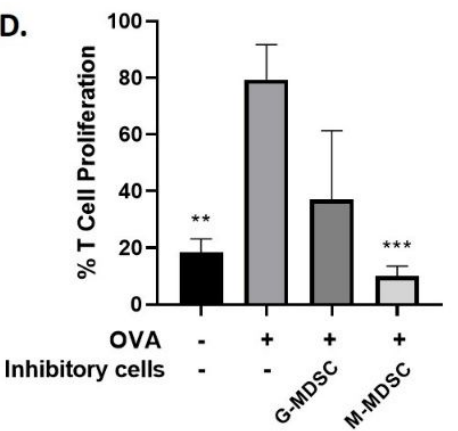

Supplementary figure S14. Analysis of myeloid derived suppressor cells (MDSC) by flow cytometry. A. Peritoneal cavity (PC) cells were stained with fluorophore conjugated anti-CD11b, Ly6C and Ly6G and gated for live and CD11 $\mathrm{b}^{+}$cells to display in Ly6C and Ly6G contour plots. The percentage of the four populations of the cells shown in Q1, Q2, Q3 and Q4 is indicated on the upper left corner of each plot (blue number). \% of MDSC is the sum of Q1+Q2+Q4. One representative experiment is shown for naïve mice, PBS-treated tumor-bearing mice, and RNA$\mathrm{OG}$ treated mice with tumor regression. B. Flow cytometry was used to analyze granulocytic MDSC (G-MDSC) and monocytic MDSC (M-MDSC) isolated from PC cells of tumor-bearing mice by MDSC-MACS columns. Percentage of each MDSC population and the purity after isolation is indicated in the boxes. C. Splenic OVA-specific transgenic CD4 T cells were stained with Cytopainter proliferative dye and cultured with bone marrow cells (BMC) in the presence of OVA, with or without MDSCs. Proliferation was determined by the dilution of Cytopainter and shown in the brackets. D. Summary of T cell proliferation, measured by the dilution of the proliferative dye, was displayed as the mean $\pm \mathrm{SD}(\mathrm{n}=3)$. ${ }^{* * *}$ and $* *$ indicates $\mathrm{p}<0.001$ and $\mathrm{p}<0.01$, respectively. 